\title{
Changes in factor VII-activating protease in a bleomycin-induced lung injury rat model and its influence on human pulmonary fibroblasts in vitro
}

\author{
EN MU, XIUJUAN LIU, SONG CHEN, LINLIN ZHI, XIN LI, XIANGHUA XU and XIAOCHUN MA
}

\author{
Department of Intensive Care Medicine, The First Affiliated Hospital \\ of China Medical University, Shenyang 110001, P.R. China
}

Received May 6, 2010; Accepted June 21, 2010

DOI: 10.3892/ijmm_00000498

\begin{abstract}
Acute lung injury (ALI) and acute respiratory distress syndrome (ARDS) are characterized by non-cardiogenic pulmonary edema, respiratory distress and hypoxemia. Factor VII-activating protease (FSAP) is a plasma-derived protease which inhibits the proliferation and migration of hepatic stellate cells and vascular smooth muscle cells. This study was designed to determine the dynamic expression changes in FSAP in a bleomycin-induced pulmonary fibrosis rat model and the influence of FSAP on human pulmonary fibroblasts (HPF). The expression of FSAP was examined in a rat model of bleomycin-induced pulmonary fibrosis by immunohistochemical staining, quantitative real-time RT-PCR and Western blot analysis. The influence of FSAP on cell proliferation and migration of HPF was investigated by the BrdU incorporation assay and transwell cell culture chambers, respectively. The effect of FSAP on platelet-derived growth factor (PDGF)-stimulated p42/p44 mitogen-activated protein kinase (MAPK) activation on HPF was determined by Western blot analysis. FSAP was observed prominently in alveolar epithelial cells as well as microvascular endothelial cells of the lung parenchyma and was markedly increased at the early phase of bleomycin-induced pulmonary fibrosis, but decreased at the late stage, particularly during the pulmonary fibrosis. FSAP inhibited PDGF-stimulated proliferation, migration, p42/p44 MAPK phosphorylation and collagen III synthesis of the HPF. We concluded that epithelial cells in lungs represent a source of FSAP at the early stage in acutely injured lung. Moreover, we demonstrated an inhibitory effect of FSAP on PDGF-stimulated proliferation and migration of HPF in vitro, suggesting that FSAP may modulate inflammation and exert a beneficial effect on ARDS.
\end{abstract}

Correspondence to: Dr Xiaochun Ma, Department of Intensive Care Medicine, The First Affiliated Hospital of China Medical University, 155 Nanjing North Street, Shenyang 110001, P.R. China E-mail: xiaochun_ma@163.com

Key words: factor VII-activating protease, bleomycin, pulmonary fibrosis

\section{Introduction}

Acute lung injury (ALI) and its more severe form, acute respiratory distress syndrome (ARDS), are common devastating clinical syndromes which are characterized by non-cardiogenic pulmonary edema, respiratory distress and hypoxemia (1). Although the mortality rate associated with ARDS has improved in the last decade, $40-70 \%$ of patients still die from this syndrome, and survivors encounter significant physical and psychological impairments (2). ALI/ARDS are caused by a number of processes that direct or indirect injury to the lung (3). The traditional model of ARDS suggests that lung injury follows an orderly sequential pattern in which damage to the alveolar capillary membrane accompanied by edema formation is followed by active clearance of edema fluid and subsequent repair of the alveolar-capillary membrane with a varying degree of fibrosis (4). It is now recognized that these processes occur simultaneously in the lungs of patients with ARDS (5). Previous studies have indicated that changes in various cytokines such as platelet-derived growth factor (PDGF) and transforming growth factor (TGF) are involved in these processes $(6,7)$. Therefore, understanding the changes in the cytokines in these processes may provide possible opportunities for therapeutic intervention of ARDS.

Factor VII-activating protease (FSAP) is a plasma-derived protease structurally homologous to members of the hemostasis family $(8,9)$. It serves to activate pro-urokinase as well as factor VII and might play a role in the regulation of both coagulation and fibrinolysis (9). Most recently, Mambetsariev and colleagues found elevated expression of FSAP (termed hyaluronic acid binding protein 2, HABP2) in the endothelium of a lipopolysaccharide (LPS)-induced ALI model. FSAP negatively regulated vascular integrity via activation of protease-activated receptor/RhoA/Rho kinase signaling, contributing to the disruption of the endothelial cell barrier in ALI (10). In addition, Wygrecka et al reported that the FSAP protein level and activity were markedly increased in the plasma and bronchoalveolar lavage (BAL) fluid of patients with ARDS, and FSAP was observed in alveolar macrophages, bronchial epithelial and endothelial cells of lungs of patients with ARDS, but was restricted to alveolar macrophages in the controls. Furthermore, the mRNA expression of FSAP was induced by LPS and interleukin- 8 in human lung microvascular endothelial cells and in bronchial epithelial cells 
(11). These novel findings strongly suggest that the expression level and altered cellular expression of FSAP may play a vital role in the pathogenesis of ARDS. Nonetheless, the precise role of FSAP in ARDS needs to be further elucidated.

Accumulating evidence indicates that basic fibroblast growth factor (bFGF) and platelet-derived growth factor-BB (PDGF-BB) participate in the pathogenesis of ARDS by induction of endothelial cell activation, stimulation of fibroblast proliferation as well as the promotion of pulmonary fibrosis $(12,13)$. Moreover, FSAP inhibits the proliferation and migration of hepatic stellate cells and vascular smooth muscle cells by cleavage of PDGF-BB, and thereby functions as a suppressor of fibrosis and inflammation response (14). These findings led us to explore whether similar mechanisms of FSAP also play a role in the progression of ARDS. Additionally, since pulmonary fibrosis is a long-term dynamic and progressive disease, the investigation of changing levels of FSAP along a time course may have significance in understanding the real pathophysiology of this condition. To this end, in the present study we investigated the expression of FSAP in bleomycin-induced acute lung injury and fibrosis in a rat model. The influence of FSAP on cultured fibroblasts was also further examined.

\section{Materials and methods}

Animals and reagents. Pathogen-free male Sprague-Dawley rats $(200 \pm 20 \mathrm{~g})$ were purchased from the Experimental Animal Center of China Medical University. Bleomycin was obtained from Nippon Kayaku Co., Ltd. (Tokyo, Japan), and the FSAP recombinant protein was purchased from Abnova (Taiwan, China).

Preparation and characterization of antibodies against FSAP. A rabbit polyclonal antibody against FSAP was prepared and characterized by Cowin Biotech (Beijing, China). In brief, the FSAP recombinant protein emulsified in an equal volume of Freund's complete adjuvant (FCA) was administered to each rabbit in the form of mutifocal intradermal injections (first immunization). Two booster injections were performed as above with Freund's incomplete adjuvant (FIA) substituted for FCA at 21 and 35 days after the first immunization, respectively. Three days after the third injection, blood samples were collected from the marginal ear vein of the rabbits to test the titer using indirect ELISA. Then, the blood sample with high titer was purified using protein A affinity chromatography to obtain the antibodies. The titer of the antibodies was detected with indirect ELISA.

Bleomycin-induced pulmonary fibrosis model. The animal study protocol was approved by the Ethics Committee for Animal Experiments of the China Medical University, Shenyang, China. Forty rats were randomly divided into the control group $(n=20)$ and the bleomycin-administered experimental group $(n=20)$. Each rat was anesthetized with sodium pentobarbital $(100 \mathrm{mg} / \mathrm{kg})$ and subjected to a tracheostomy. The rats in the experimental groups received intratracheal instillation of $4 \%$ bleomycin $(5 \mathrm{mg} / \mathrm{kg})$. Upright spin was then used to ensure a homogeneous distribution of bleomycin to the rat lungs. The control animals received intratracheal saline only. Five rats from the experimental group and 5 from the control group were sacrificed at 3, 7, 14 and 28 days after bleomycin instillation.

Histopathology. The middle lobes of the right lungs were embedded in paraffin, following fixation in $10 \%$ buffering formalin, and subsequently hematoxylin and eosin (H\&E) staining was performed using 4-mm sections to evaluate the degree of pulmonary fibrosis.

Immunohistochemical staining. Following deparaffinization of the sections, streptavidin-biotinylated peroxidase immunohistochemical staining was performed to detect the expression of FSAP, as described elsewhere. A rabbit polyclonal antibody against rat FSAP (dilution 1:500) was used as primary antibody.

Cell culture. Human pulmonary fibroblasts (HPF) were obtained from Bioleaf Biotechnology Co., Ltd. (Shanghai, China). The cells were maintained in Dulbecco's modified Eagle's medium (DMEM, Gibco, Grand Island, NY, USA) supplemented with fetal bovine serum (FBS) and cultured at $37^{\circ} \mathrm{C}$ in a $5 \% \mathrm{CO}_{2}$ incubator. The cells were routinely passaged, and cells at logarithmic growth phase were used for the experiments.

Quantitative real-time $R T$-PCR. Total RNA from the lung tissues and the treated HPF was isolated using Trizol reagent (Life Technologies, Inc., Rockville, MD) according to the manufacturer's instructions. Reverse transcription was performed on $1 \mu \mathrm{g}$ of total RNA from each sample. Quantitative real-time RT-PCR was performed using SYBR Green on an Exicycler ${ }^{\mathrm{TM}} 96$ Real-Time Quantitative Thermal Block (Bioneer, Daejeon, Korea). The PCR primer sequences were designed according to the FSAP and COL3A1 gene sequences reported in GenBank and were chemically synthesized: FSAP, forward 5'-CTCCTGGATGCCAAAGT CAA-3'; reverse 5'-GTAGGTGCCGTCCTTCTCAC-3'; COL3A1, forward 5'-CCACCCTGAACTCAAGAGC-3'; reverse 5'-TGAACTGAAAGCCACCATT-3'. The specificity of the PCR was confirmed by examining the dissociation reaction plot subsequent to real-time RT-PCR. $\beta$-actin served as the constitutive control. PCR reactions of each sample were conducted in triplicate. Data were analyzed through the comparative threshold cycle $\left(\mathrm{C}_{\mathrm{T}}\right)$ method.

Western blot analysis. Total proteins from the lung tissues and the treated HPF were separated on SDS-polyacrylamide gels and then electro-transferred to PVDF membranes. FSAP and COL3A1 proteins were visualised by immunodetection using a rabbit polyclonal FSAP antibody (Cowin Biotech, Beijing, China) or a rabbit polyclonal COL3A1 antibody (Santa Cruz Biotechnology, CA, USA). After subsequent incubation with horseradish peroxidase-linked secondary antibodies the labeled proteins were detected using an enhanced chemiluminescence (ECL) kit (AmershamPharmacia, Freiburg, Germany).

Bromodeoxyuridine (BrdU) incorporation. Cell proliferation was evaluated by using BrdU incorporation assays. HPF 

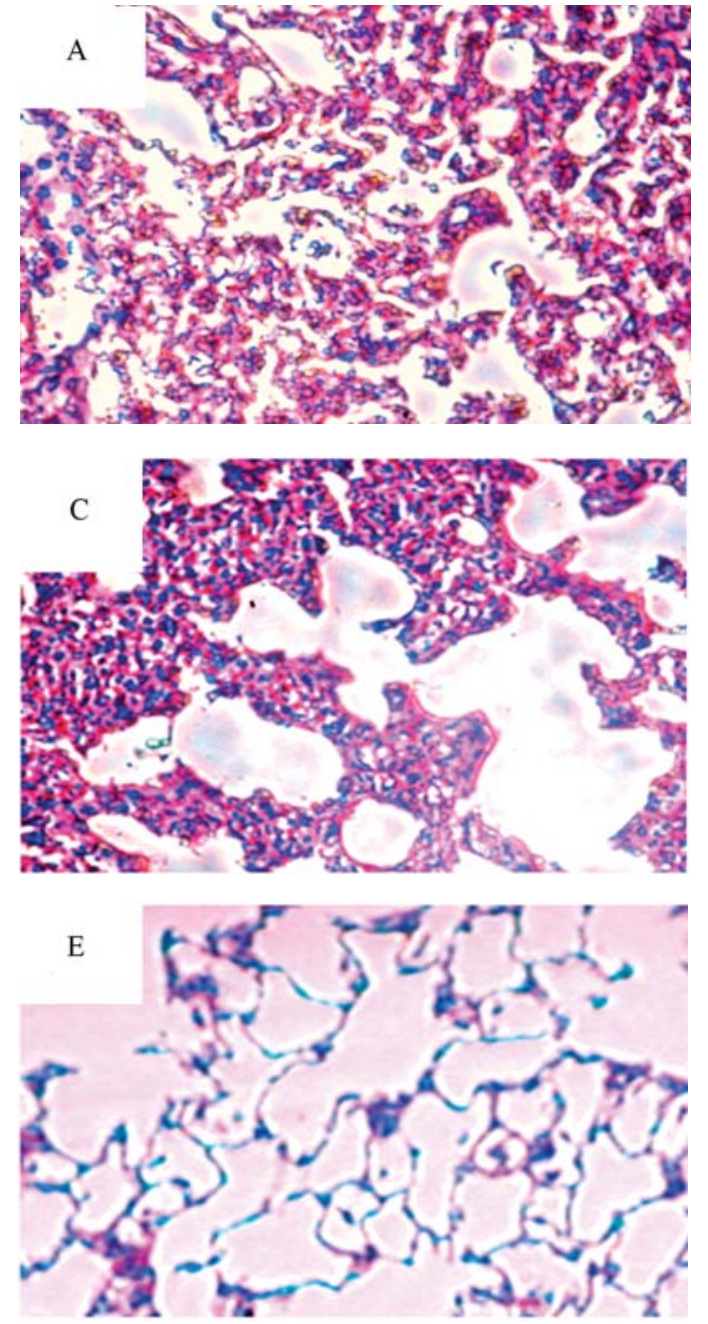

Figure 1. A histopathological time course of pulmonary fibrosis induced by bleomycin (original magnification $\times 200$ ). (A) On day 3, a prominent inflammatory reaction was noted, showing congestion of small blood vessels, minor hemorrhage in interstitial and alveolar space, and effusion of neutrophils as well as occasional widening of the alveolar septum. (B) On day 7 thickening of alveolar walls and intraalveolar edema became prominent More neutrophils and macrophages were present and inflammatory cell infiltration spread to the interstitial and alveolar spaces. Diminishing of alveolar space and proliferation of blood capillaries were strongly induced (C) On day 14, marked widening of alveolar septum was observed and the collagen fibers became apparent and widened, accompanied by an increase in lymphocytes and fibroblasts. (D) On day 28, the level of fibrosis further progressed. Abundant collagen fiber deposition and collapse of alveolar spaces were observed, along with marked thickening of capillary walls. (E) In the saline-treated control animals, such morphological changes were not observed at any time point examined.

were seeded in a 96-well plate $\left(2 \times 10^{4}\right.$ cells per well), and BrdU (Nippon Kayaku Co., Ltd., Tokyo, Japan) was added to the medium, cells were grown for $24 \mathrm{~h}$, and the medium was changed to fresh medium without serum. After an additional $24 \mathrm{~h}$, cells were treated with the appropriate agonist mixtures as indicated in the appropriate figures or figure legends. Forty-eight hours post treatment, BrdU incorporation assays were carried out.

Cell migration assay. In vitro migration assays were conducted in a 24-well plate applying transwell cell culture

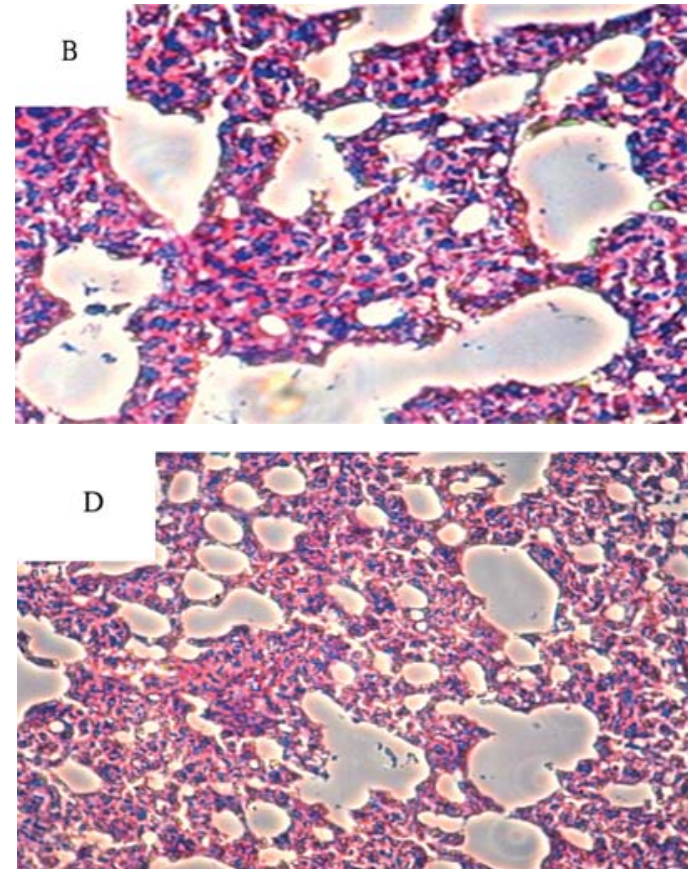

chambers. HPF $\left(1 \times 10^{5}\right)$ were seeded in the upper chamber containing DMEM with $1 \%$ FBS and $10 \mathrm{~g} / 1$ BSA. Heparin $(10 \mu \mathrm{g} / \mathrm{ml})$, PDGF-BB $(20 \mathrm{ng} / \mathrm{ml})$, FSAP $(12 \mu \mathrm{g} / \mathrm{ml})$ or aprotinin $(10 \mu \mathrm{g} / \mathrm{ml})$ was added to the lower chamber as described in the appropriate figures or figure legends. After the cells were incubated for $16 \mathrm{~h}$ at $37^{\circ} \mathrm{C}$ with $5 \% \mathrm{CO}_{2}$, the insert was washed with PBS, and cells on the top surface of the insert were removed. Migrated cells were fixed, stained and counted in five randomly (x400) selected fields with an inverted microscope, and the average numbers of cells per field were counted.

Phosphorylation of mitogen-activated protein kinase (MAPK) $p 42 / p 44$. HPF were serum starved overnight and stimulated for $15 \mathrm{~min}$ with the appropriate agonist mixtures that were pre-incubated for $60 \mathrm{~min}$ at $37^{\circ} \mathrm{C}$. Subsequently, total proteins from treated cells were processed for Western blot analysis as described above. Phosphorylated forms of MAPK p42 and p44 (MAPK-p42/p44) were detected with an antibody against phosphoMAPK-p42/p44, and the total MAPK was also determined to show equal amounts of total cellular MAPK in all test conditions (both from Cell Signaling Technology, Beverly, MA, USA).

Statistical analysis. All data were shown as means \pm SD. Analysis of data from multiple groups was performed by ANOVA using SPSS 13.0 software. A P-value $<0.05$ was considered to be statistically significant.

\section{Results}

Sequential histopathological changes in bleomycin-induced pulmonary fibrosis. Three, 7, 14 and 28 days after the rats were instilled with bleomycin, H\&E staining was performed to evaluate the histopathological changes in bleomycininduced pulmonary fibrosis. As shown in Fig. 1A, in the bleomycin-treated experimental group, a prominent 

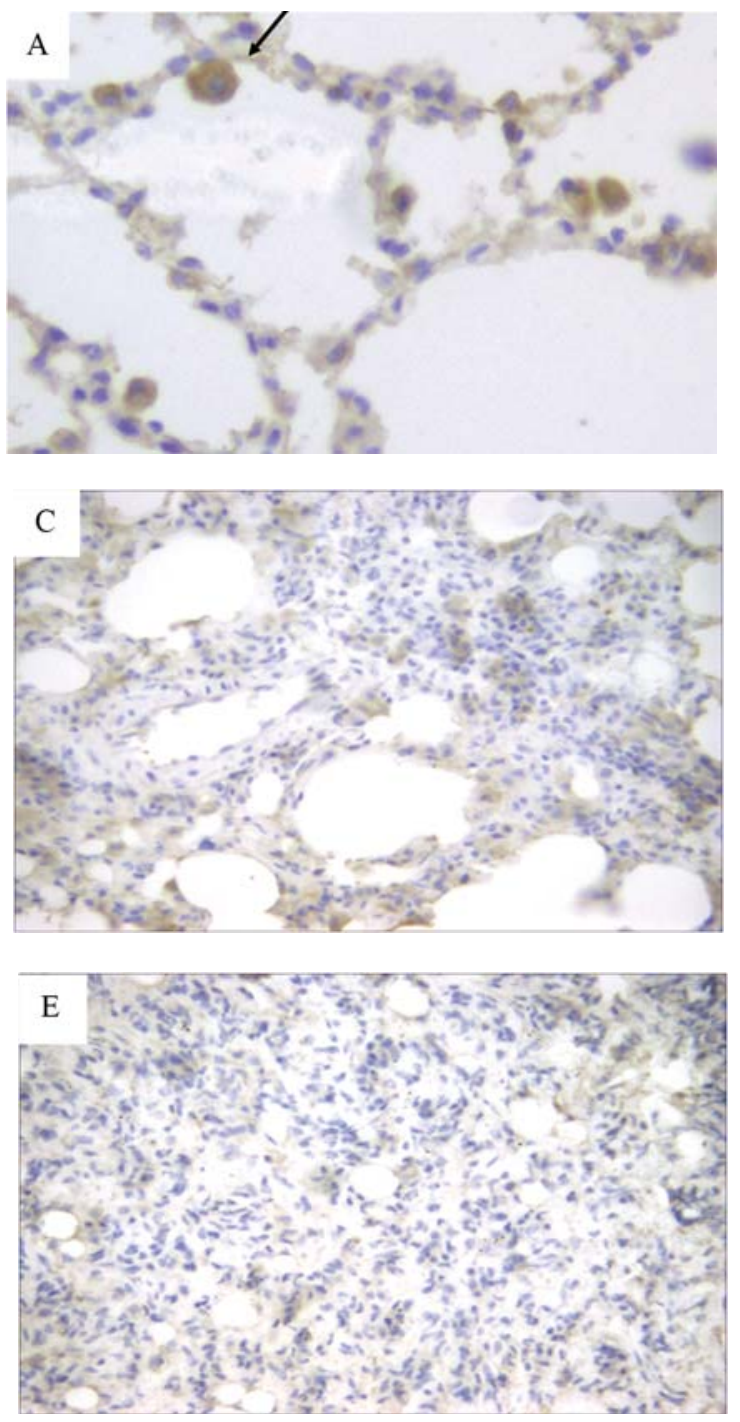
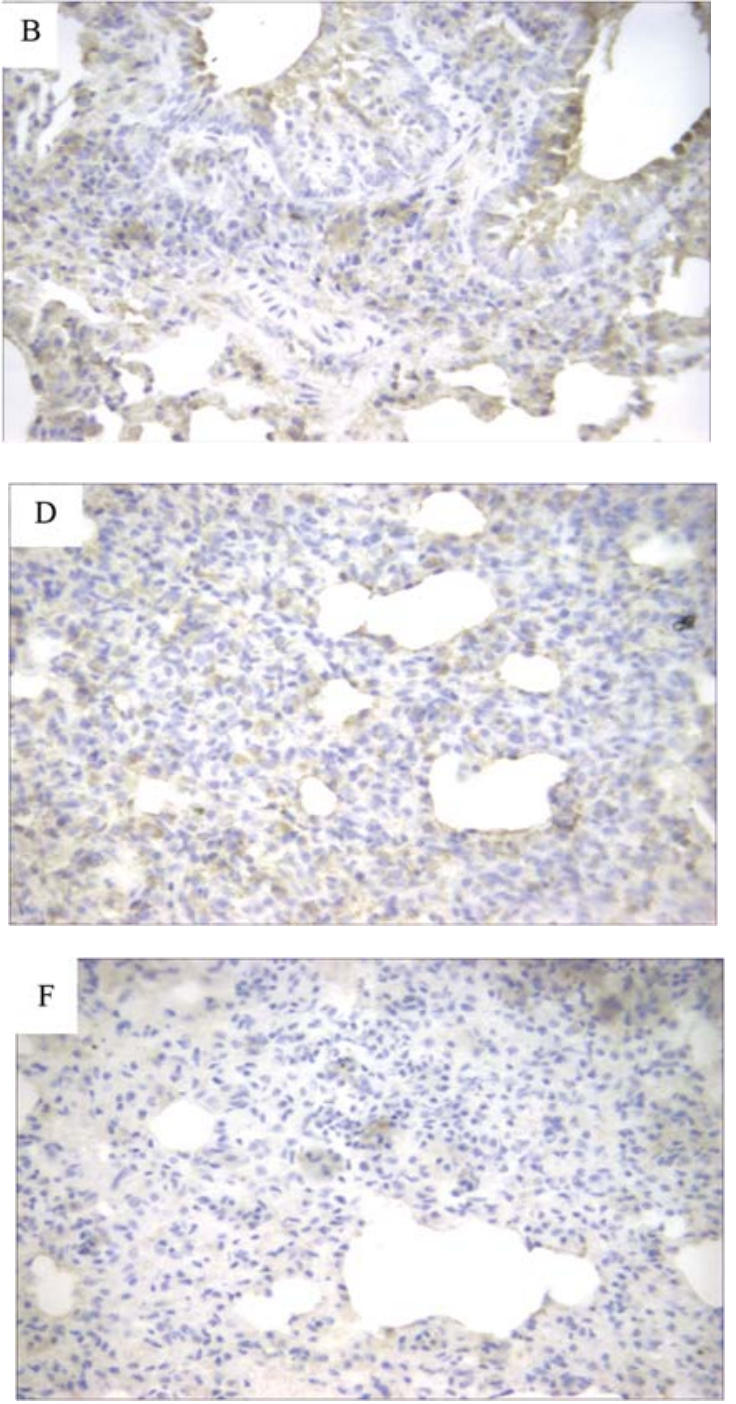

Figure 2. Time-course changes in factor VII-activating protease (FSAP) localization in bleomycin-induced pulmonary fibrosis. Representative immunohistochemical results of FSAP in the saline-treated control animals (A) and in the bleomycin-treated group on days 3, 7, 14, and 28 after bleomycin administration (B-F) (original magnification x200). (A) In the saline-treated control group, positive signals of FSAP were observed weakly in a small number of alveolar macrophages. On day 3, positive signals for FSAP were observed prominently in alveolar epithelial cells (B) and microvascular endothelial cells (C). (D and E) On day 7 and 14, no obvious change in FSAP expression and localization was observed. (F) Positive signals of FSAP were markedly diminished on day 28.

inflammatory reaction was noted from the third day, showing congestion of small blood vessels, minor hemorrhage in interstitial and alveolar space, and effusion of neutrophils as well as occasional widening of the alveolar septum. On day 7 , thickening of alveolar walls and intraalveolar edema became prominent. More neutrophils and macrophages were present, and inflammatory cell infiltration spread to the interstitial and alveolar spaces. A decrease in the alveolar space and proliferation of blood capillaries were strongly induced. Additionally, the number of fibroblasts was markedly increased (Fig. 1B). As shown in Fig. 1C, the number of infiltrating macrophages peaked on day 14 . In contrast, a marked widening of the alveolar septum was observed, and the collagen fibers became apparent and widened, accompanied by an increase in lymphocytes and fibroblasts. On day 28 , the level of fibrosis further progressed. Abundant collagen fiber deposition and collapse of alveolar spaces were observed, along with a marked thickening of capillary walls (Fig. 1D).
In the saline-treated control animals, such morphological changes were not observed at any time point examined (Fig. 1E).

Time-course changes in FSAP expression and localization in bleomycin-induced pulmonary fibrosis. Immunohistochemical staining was employed to examine the changes in expression and localization during bleomycin-induced pulmonary fibrosis. In the saline-treated control animals, positive signals of FSAP were observed weakly in a small number of alveolar macrophages, but was absent in infiltrating monocytes and granulocytes (Fig. 2A). On day 3 after bleomycin instillation, positive signals for FSAP were observed prominently in alveolar epithelial cells and microvascular endothelial cells. At the later time points, no obvious changes in FSAP expression and localization were observed on day 7 and 14, whereas positive signals of FSAP were markedly diminished on day 28 (Fig. 2B-F). 
A

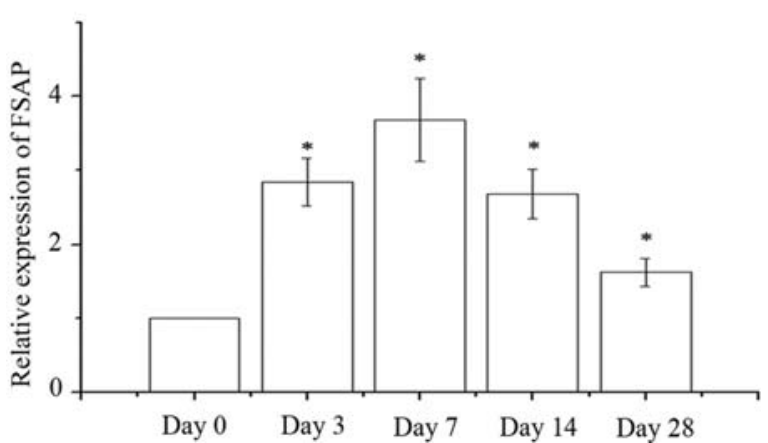

B

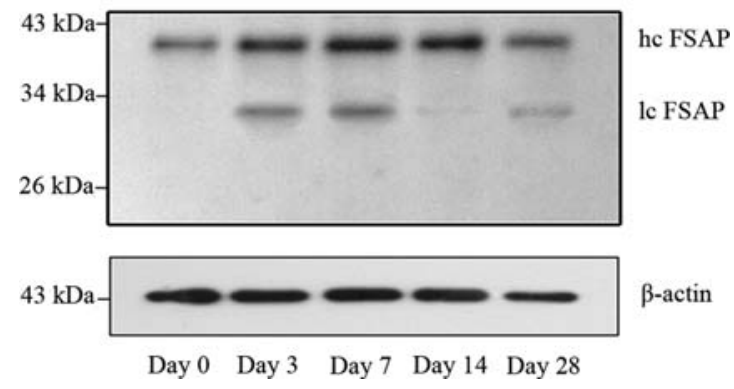

Figure 3. Time-course changes in factor VII-activating protease (FSAP) expression in the lung parenchyma of the bleomycin-treated group shown by quantitative real-time RT-PCR (A) and Western blot analysis (B), respectively. (A) According to quantitative real-time RT-PCR, mRNA expression of FSAP tended to increase at the beginning of bleomycin instillation, then peaked on day 7 , and deceased thereafter. ${ }^{*} \mathrm{P}<0.05$ vs control. (B) FSAP protein levels in the above-mentioned group were consistent with mRNA expression by Western blotting. Representative blots are shown, and protein size is expressed in kDa. hc FSAP, heavy chain of proteolytically active two-chain FSAP; lc FSAP, light chain of proteolytically active two-chain FSAP.

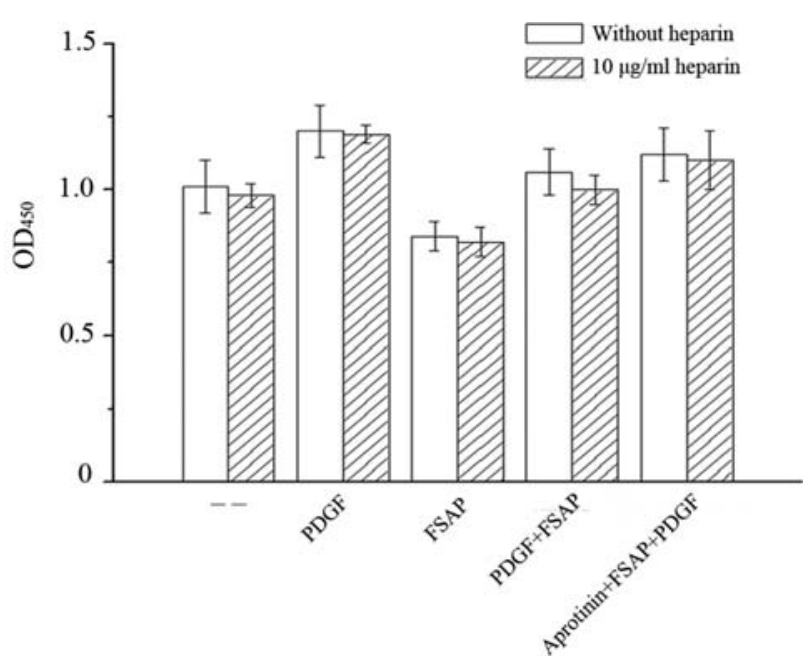

Figure 4. Effect of factor VII-activating protease (FSAP) on proliferation of human pulmonary fibroblasts (HPF). HPF were grown in 96-well plates and were serum starved overnight. FSAP, platelet-derived growth factor (PDGF)-BB and/or heparin and/or aprotinin were added to the wells. Fortyeight hours post treatment, BrdU incorporation assays were carried out.

In order to further determine the expression level of FSAP in bleomycin-induced pulmonary fibrosis, FSAP mRNA and protein levels were measured by quantitative real-time RT-PCR and Western blot analysis, respectively. As shown in Fig. 3A, the mRNA expression of FSAP tended to increase at the beginning of bleomycin instillation, then peaked on day 7 , and deceased thereafter. Meanwhile, changes in the Western blotting were in accordance with the findings in the quantitative real-time RT-PCR study (Fig. 3B). These results confirmed that the expression level of FSAP was markedly increased before the progression of pulmonary fibrosis, but decreased during pulmonary fibrosis.

FSAP inhibits DNA synthesis, cell proliferation and migration in $H P F$. The influence of FSAP on cell proliferation and migration in HPF was investigated by BrdU incorporation and transwell cell culture chambers, respectively. As shown in Fig. 4, cell proliferation was stimulated by both PDGF-BB

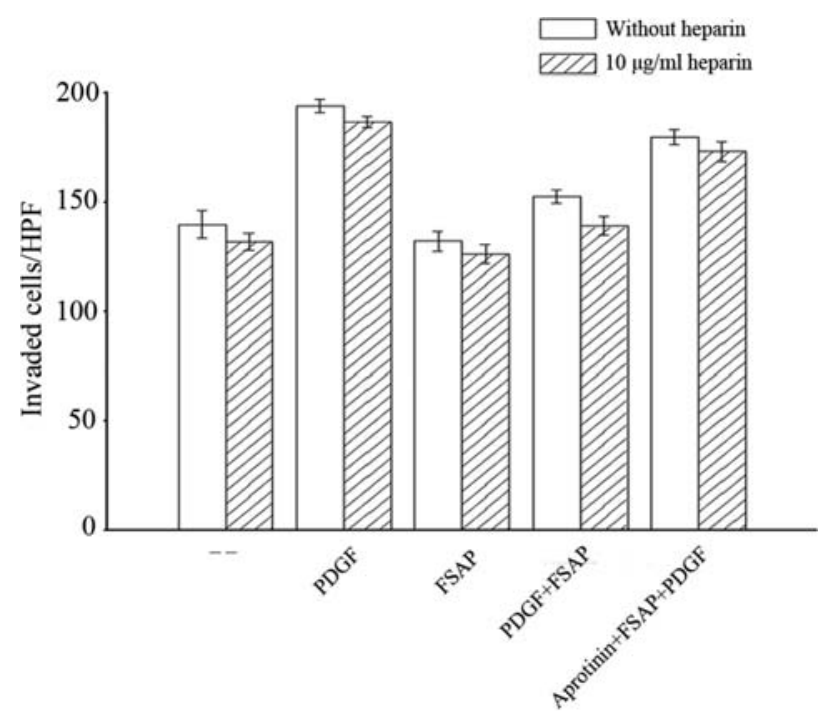

Figure 5. Effect of factor VII-activating protease (FSAP) on migration of human pulmonary fibroblasts (HPF). HPF were seeded in the upper chamber. FSAP, platelet-derived growth factor (PDGF)-BB and/or heparin and/or aprotinin were added to the lower chamber.

and heparin. Although FSAP alone did not have a significant inhibitory effect on cell proliferation, when FSAP and PDGF-BB were incubated in the presence of heparin there was a loss of PDGF activity. In the presence of aprotinin, which inhibits the proteolytic activity of FSAP, there was no inhibition of PDGF-BB stimulation.

As shown in Fig. 5, PDGF-BB and heparin, not only influenced the proliferation of HPF but also cell migration. FSAP had an inhibitory effect on the migration of HPF. Particularly in the presence of heparin, a strong inhibitory effect of FSAP was observed, and this effect was reversed by the presence of FSAP enzymatic activity blocking aprotinin.

Effect of FSAP on PDGF-BB-stimulated phosphorylation in $H P F$. PDGF-BB is a stimulator of MAPK-p42/44 phosphorylation in HPF. FSAP itself did not inhibit the effect of PDGF-BB on MAPK phosphorylation, but in the presence of 
A

\begin{tabular}{|c|c|c|c|c|c|c|c|}
\hline Heparin & - & - & - & - & + & + & + \\
\hline PDGF & - & - & + & + & - & - & + \\
\hline FSAP & - & + & - & + & - & + & - \\
\hline
\end{tabular}
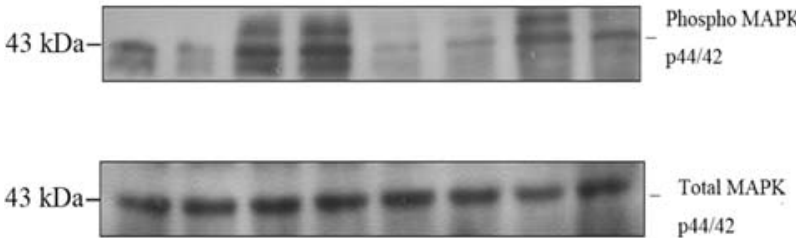

B

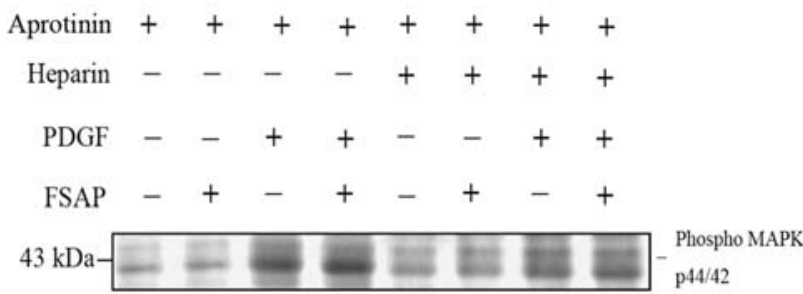

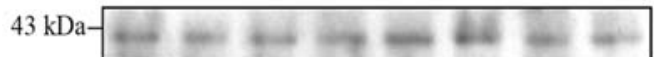

Total MAPK $\mathrm{p} 44 / 42$

Figure 6. Effect of factor VII-activating protease (FSAP) on mitogen-activated protein kinase (MAPK) phosphorylation in human pulmonary fibroblasts (HPF). (A) HPF were serum starved overnight. FSAP, platelet-derived growth factor (PDGF)-BB and/or heparin were pre-incubated for 60 min at $37^{\circ} \mathrm{C}$ and then added to the cells for $15 \mathrm{~min}$. Thereafter, the cells were lysed and used for Western blot analysis. (B) The inhibitory effect of aprotinin on FSAPmediated inhibition was determined.

A
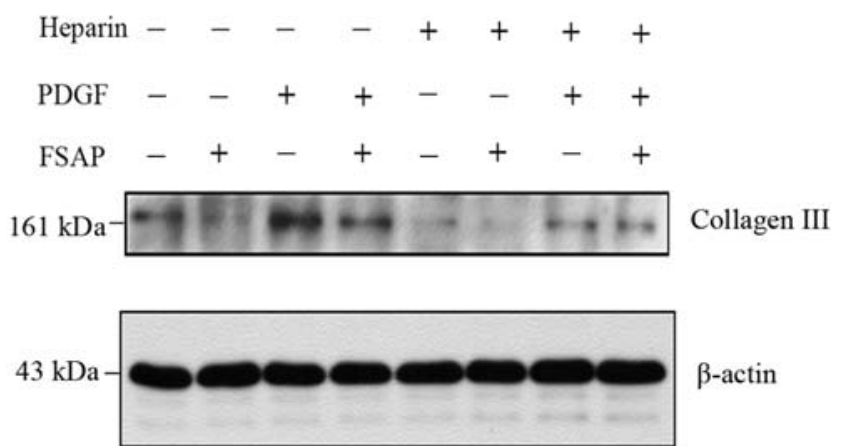

B

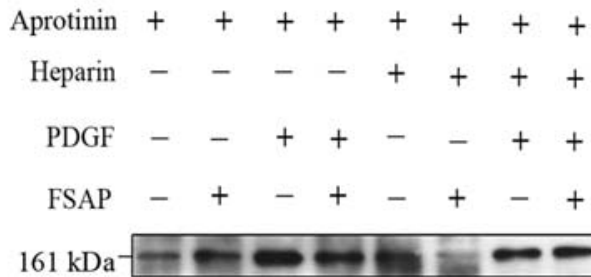

Collagen III

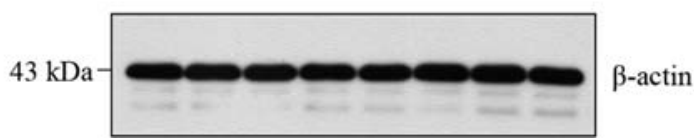

Figure 7. Effect of factor VII-activating protease (FSAP) on the synthesis of collagen III in human pulmonary fibroblasts (HPF). (A) HPF were serum starved overnight. FSAP, platelet-derived growth factor (PDGF)-BB and/or heparin were pre-incubated for 60 min at $37^{\circ} \mathrm{C}$ and were added to the cells for $48 \mathrm{~h}$. Thereafter, the cells were lysed and used for Western blot analysis. (B) The inhibitory effect of aprotinin on FSAP-mediated inhibition was also determined.

heparin, a strong inhibitory effect of FSAP was induced. Similarly, this effect was also reversed by the presence of aprotinin (Fig. 6A and B).

Effect of FSAP on collagen III synthesis in HPF. HPF, when incubated with PDGF-BB, showed markedly increased mRNA and protein levels of collagen III, as shown in Fig. 7, FSAP and heparin alone had a slight inhibitory effect on the synthesis of collagen III. Notably, when FSAP and PDGFBB were incubated in the presence of heparin, a PDGF-BBmediated stimulus effect was also decreased by FSAP. In accordance with the above results, there was a loss of the FSAP-mediated effect in the presence of aprotinin.

\section{Discussion}

In the present study, the expression level of FSAP was examined in a bleomycin-induced pulmonary fibrosis rat model along a time course. We showed that FSAP was markedly increased in the lung parenchyma at the early phase of bleomycin-induced pulmonary fibrosis, but decreased at the late stage, particularly during the pulmonary fibrosis.
Furthermore, the influence of FSAP on cultured HPF was also examined. We demonstrated an inhibitory effect of FSAP on PDGF-stimulated proliferation and migration of HPF in vitro. Collectively, these observations strongly suggest that FSAP might be associated with the development of ARDS.

FSAP protein and activity levels have previously been shown to be increased in the plasma and BAL fluid of patients with ARDS (11). However, it is essential to further investigate the dynamic changes in FSAP expression and localization in pulmonary fibrosis, which will facilitate our understanding of the precise role of FSAP in ARDS. Bleomycin-induced pulmonary fibrosis is considered to be the most common and acceptable model (15). In the present study, we found that FSAP was elevated at an early stage, but subsequently decreased at a late stage in a bleomycin-induced pulmonary fibrosis rat model. In addition, the expression of FSAP was prominently observed in alveolar epithelial cells and microvascular endothelial cells. This is consistent with a previous study showing that FSAP was observed in these cells in the lungs of patients with ARDS (11). Thus, these findings indicated that epithelial cells in lungs represent a 
source of FSAP in ARDS. It therefore seems reasonable to propose that FSAP has a critical, harmful or protective role in ARDS.

Another major finding of our study is that an inhibitory action of FSAP on PDGF-stimulated proliferation and migration of HPF, which is similar to the effect of FSAP on hepatic stellate cells and vascular smooth muscle cells in prior studies, was noted $(14,16)$. In addition, the enzymatic activity of FSAP was required for the inhibition of proliferation and migration as the effects of FSAP were inhibited by aprotinin. From our results, it is clear that inhibition by FSAP was only apparent in the presence of heparin, which further confirmed the cleavage of the PDGF-B chain in the receptor activation domain by FSAP. However, some concerns involving FSAP need to be determined. For instance, previous studies have indicated that FSAP can either diminish or promote the activity of the bFGF in a cell-specific manner (17-19). Whether similar interactions between FSAP and other cytokines also occur in HPF should be addressed.

Collagen accumulation is a common hallmark of ARDS and increased levels of collagen, particularly collagen I and collagen III, are present in the lungs of patients with acute lung injury $(20,21)$. FSAP has been demonstrated to inhibit wire injury-induced neointimal lesion formation in the mouse, and this anti-inflammatory effect of FSAP was mediated by inhibiting the proliferation and migration of vascular smooth muscle cells and monocytes/macrophages in the neointima of the FSAP-treated vessels (22). Herein, we observed that the PDGF-stimulated synthesis of "collagen III was inhibited by FSAP. Keeping in mind all of our observations, it is reasonable to conclude that an increased level of FSAP, as observed in the present study, may modulate inflammation and exert a beneficial effect at the early stage of acutely injured lung, whereas a decreased level of FSAP at the late stage may promote pulmonary fibrosis by stimulating the synthesis of collagens. Nonetheless, the detailed mechanisms underlying the dysregulation of FSAP in ARDS need to be clarified.

In summary, we demonstrated an inhibitory effect of FSAP on PDGF-stimulated proliferation and migration of HPF in vitro. In addition, the dynamic changes in FSAP expression in a bleomycin-induced pulmonary fibrosis rat model indicate that FSAP may modulate inflammation and exert a beneficial effect on ARDS, suggesting that exogenous administration of FSAP may serve as a potential strategy for therapeutic interventions of ARDS.

\section{References}

1. Gao L and Barnes KC: Recent advances in genetic predisposition to clinical acute lung injury. Am J Physiol Lung Cell Mol Physiol 296: L713-L725, 2009.

2. Rubenfeld GD, Caldwell E, Peabody E, et al: Incidence and outcomes of acute lung injury. N Engl J Med 353: 1685-1693, 2005.

3. Pietropaoli A and Georas SN: Resolving lung injury: a new role for Tregs in controlling the innate immune response. J Clin Invest 119: 2891-2894, 2009.
4. Marshall R, Bellingan G and Laurent G: The acute respiratory distress syndrome: fibrosis in the fast lane. Thorax 53: 815-817, 1998.

5. Chesnutt AN, Matthay MA, Tibayan FA and Clark JG: Early detection of type III procollagen peptide in acute lung injury. Pathogenetic and prognostic significance. Am J Respir Crit Care Med 156: 840-845, 1997.

6. Zagai U, Fredriksson K, Rennard SI, Lundahl J and Skold CM: Platelets stimulate fibroblast-mediated contraction of collagen gels. Respir Res 4: 13, 2003.

7. Budinger GR, Chandel NS, Donnelly HK, Eisenbart J, Oberoi M and Jain M: Active transforming growth factor-betal activates the procollagen I promoter in patients with acute lung injury. Intensive Care Med 31: 121-128, 2005.

8. Romisch J, Feussner A, Vermohlen S and Stohr HA: A protease isolated from human plasma activating factor VII independent of tissue factor. Blood Coagul Fibrinolysis 10: 471-479, 1999.

9. Romisch J: Factor VII activating protease (FSAP): a novel protease in hemostasis. Biol Chem 383: 1119-1124, 2002.

10. Mambetsariev N, Mirzapoiazova T, Mambetsariev B, et al: Hyaluronic acid binding protein 2 is a novel regulator of vascular integrity. Arterioscler Thromb Vasc Biol 30: 483-490, 2010.

11. Wygrecka M, Markart P, Fink L, Guenther A and Preissner KT: Raised protein levels and altered cellular expression of factor VII activating protease (FSAP) in the lungs of patients with acute respiratory distress syndrome (ARDS). Thorax 62: 880-888, 2007.

12. Henke C, Marineili W, Jessurun J, et al: Macrophage production of basic fibroblast growth factor in the fibroproliferative disorder of alveolar fibrosis after lung injury. Am J Pathol 143: 1189-1199, 1993.

13. Madtes DK, Busby HK, Strandjord TP and Clark JG: Expression of transforming growth factor-alpha and epidermal growth factor receptor is increased following bleomycin-induced lung injury in rats. Am J Respir Cell Mol Biol 11: 540-551, 1994.

14. Roderfeld M, Weiskirchen R, Atanasova S, et al: Altered factor VII activating protease expression in murine hepatic fibrosis and its influence on hepatic stellate cells. Liver Int 29: 686-691, 2009.

15. Gabazza EC, Taguchi O and Adachi Y: Bleomycin-induced lung fibrosis: the authors should have used another method to induce pulmonary lesions resembling human idiopathic pulmonary fibrosis. Am J Respir Crit Care Med 165: 845-846, 2002.

16. Kannemeier C, Al-Fakhri N, Preissner KT and Kanse SM: Factor VII-activating protease (FSAP) inhibits growth factormediated cell proliferation and migration of vascular smooth muscle cells. FASEB J 18: 728-730, 2004.

17. Etscheid M, Beer N, Kress JA, Seitz R and Dodt J: Inhibition of bFGF/EGF-dependent endothelial cell proliferation by the hyaluronan-binding protease from human plasma. Eur J Cell Biol 82: 597-604, 2004.

18. Etscheid M, Beer N and Dodt J: The hyaluronan-binding protease upregulates ERK1/2 and PI3K/Akt signalling pathways in fibroblasts and stimulates cell proliferation and migration. Cell Signal 17: 1486-1494, 2005.

19. Kress JA, Seitz R, Dodt J and Etscheid M: Induction of intracellular signalling in human endothelial cells by the hyaluronan-binding protease involves two distinct pathways. Biol Chem 387: 1275-1283, 2006.

20. Raghu G, Striker LJ, Hudson LD and Striker GE: Extracellular matrix in normal and fibrotic human lungs. Am Rev Respir Dis 131: 281-289, 1985.

21. Hagiwara S, Iwasaka H, Matsumoto S, Noguchi T and Yoshioka H: Coexpression of HSP47 gene and type I and type III collagen genes in LPS-induced pulmonary fibrosis in rats. Lung 185: 31-37, 2007.

22. Sedding D, Daniel JM, Muhl L, et al: The G534E polymorphism of the gene encoding the factor VII-activating protease is associated with cardiovascular risk due to increased neointima formation. J Exp Med 203: 2801-2807, 2006. 